\title{
Ciprofloxacin-associated seizures and acute kidney injury: a case report
}

Shi Ying Tan, MBChB, Shyh Poh Teo, MBChB, FRACP

\begin{abstract}
We herein report a case of seizures and acute kidney injury in an 85-year-old woman with a history of stroke and renal calculus who was re-admitted to hospital after ciprofloxacin treatment for Pseudomonas aeruginosa pneumonia. Caution is advised before commencing fluoroquinolone treatment in patients with a history of cerebrovascular disease and renal calculi, especially older patients or those with electrolyte disturbances, renal impairment, or neurological conditions.
\end{abstract}

Key words: Acute Kidney Injury; Drug-Related Side Effects and Adverse Reactions; Quinolones; Seizures
Department of Internal Medicine, Raja Isteri Pengiran Anak Saleha (RIPAS)

Hospital, Brunei

Both authors contributed equally

Correspondence to: Dr Shyh Poh Teo, Department of Internal Medicine, Raja Isteri Pengiran Anak Saleha (RIPAS) Hospital, Jalan Putera Al-Muhtadee Billah, Brunei Darussalam BA1710.

Email:shyhpoh.teo@moh.gov.bn

\section{CASE PRESENTATION}

In December 2018, an 85-year-old woman presented to our hospital with decompensated congestive heart failure and pneumonia. She had hypertension, hyperlipidaemia, type-2 diabetes mellitus, left renal staghorn calculus, and previous left basal ganglia haemorrhage with residual right hemiplegia. Her blood culture grew multi-sensitive Pseudomonas aeruginosa. She was initially treated with intravenous piperacillin/tazobactam, and then oral ciprofloxacin $500 \mathrm{mg}$ twice daily on discharge.

Five days later, she was readmitted to hospital after having three episodes of tonic-clonic seizures, up-rolling eyes, and facial twitching with a postictal phase. Blood test results showed acute kidney injury, with a six-fold elevation of the creatinine level from baseline. Electrolytes and glucose levels were normal. Blood, urine, and sputum cultures were negative. Urinalysis showed $\mathrm{pH}$ of $5.5,3+$ leucocytes, $2+$ protein, and $2+$ blood. Urine microscopy was unremarkable. Computed tomography of the kidneys, ureters, and bladder showed left renal parenchymal thickness with staghorn calculi similar to the previous admission, with new additional $2-3-\mathrm{cm}$ stones in the left renal pelvis. She was treated acutely with intravenous fluids, phenytoin infusion, and cessation of ciprofloxacin. Her renal function recovered, and there were no further seizures.

\section{DISCUSSION}

In this patient, it is likely that ciprofloxacin contributed to the seizures and acute kidney injury. The Naranjo adverse event score calculated was 7, which suggests that the adverse events were secondary to ciprofloxacin. ${ }^{1}$ Fluoroquinoloneinduced seizure is rare but has been documented. ${ }^{2,3}$ The antibiotic is speculated to competitively bind to gamma amino-butyric acid receptors, displacing gamma amino-butyric acid from receptors, causing a reduced seizure threshold. Fluoroquinolones may also exert agonistic effects on excitatory glutamate receptors, which increases seizure risk. ${ }^{2-4}$ Trovafloxacin has the highest epileptic potency, followed by enoxacin, lomefloxacin, moxifloxacin, ciprofloxacin, ofloxacin, and levofloxacin. ${ }^{2,3}$ Seizures usually occur between 2 and 4 days after commencement of fluoroquinolones. ${ }^{3}$

Ciprofloxacin has also been observed to induce renal failure, secondary to acute interstitial nephritis, acute tubular necrosis, crystal induced nephropathy, or rhabdomyolysis/myoglobin-associated renal failure. ${ }^{5,6}$ Acute interstitial nephritis is the most prevalent cause for ciprofloxacin-induced acute kidney injury and usually presents with fever, flank pain, skin rash, and arthralgia. In acute tubular necrosis, classically muddy brown casts are observed in urine microscopy. In the present patient, crystal 
nephropathy was suspected given the haematuria, leukocyturia, and the incidental finding of new renal 'stones' on repeat ultrasonography. This occurs more commonly with urine $\mathrm{pH}>6.8$, as ciprofloxacin is less soluble in alkaline urine. A renal biopsy is required for a definitive diagnosis. Acute kidney injury usually resolves with ciprofloxacin withdrawal and aggressive hydration.,

The pharmacokinetics of ciprofloxacin can be significantly altered in patients with impaired renal function. For patients with creatinine clearance level of $<50 \mathrm{~mL} / \mathrm{min}$ per $1.73 \mathrm{~m}^{2}$, the area under the curve of serum concentration versus time doubled, renal clearance of ciprofloxacin reduced by a quarter, and the half-life almost doubled, compared with patients with normal renal function. This suggests that the ciprofloxacin dose should be halved in those with renal impairment to achieve the same concentration. ${ }^{7}$ To maintain bacterial eradication efficacy, prolongation of ciprofloxacin administration interval is the preferred dose-adjustment approach (rather than reducing each individual drug dose). ${ }^{8}$

In patients with a history of cerebrovascular disease and renal calculi, caution is advised with fluoroquinolone treatment, particularly in older patients or those with electrolyte disturbances, renal impairment, or neurological conditions. Dose adjustment by prolongation of ciprofloxacin administration interval should be considered in those with renal impairment if fluoroquinolone therapy is necessary.

\section{DECLARATION}

The authors have no conflict of interest to disclose. No funds were obtained for production of the manuscript.

\section{REFERENCES}

1. Naranjo CA, Busto U, Sellers EM, et al. A method for estimating the probability of adverse drug reactions. Clin Pharmacol Ther 1981;30:239-45. Crossref

2. Kushner JM, Peckman HJ, Snyder CR. Seizures associated with fluoroquinolones. Ann Pharmacother 2001;35:1194-8. Crossref

3. Lahmek P, Michel L, Meunier N, Aubin HJ. A seizure attributed to ofloxacine in a woman undergoing detoxification for alcohol dependence. Case Rep Med 2009;2009:705635. Crossref

4. Akahane K, Kato M, Takayama S. Involvement of inhibitory and excitatory neurotransmitters in levofloxacin- and ciprofloxacininduced convulsions in mice. Antimicrob Agents Chemother 1993;37:1764-70. Crossref

5. Hajji M, Jebali H, Mrad A, et al. Nephrotoxicity of ciprofloxacin: five cases and a review of the literature. Drug Saf Case Rep 2018;5:17. Crossref

6. Cunha IMC, Maranzano PP, de Oliveira Rodrigues CJ. Acute kidney injury by ciprofloxacin simulating rapidly progressive glomerulonephritis. Ann Clin Case Rep 2017;2:1484.

7. Gasser TC, Ebert SC, Graversen PH, Madsen PO. Ciprofloxacin pharmacokinetics in patients with normal and impaired renal function. Antimicrob Agents Chemother 1987;31:709-12. Crossref

8. Czock D, Rasche FM. Dose adjustment of ciprofloxacin in renal failure: reduce the dose or prolong the administration interval? Eur J Med Res 2005;10:145-8. 